\title{
Realization of Translation Gateway from IPv4 to IPv6
}

\author{
Ruixia Li1, a , Yi Zeng1, b \\ ${ }^{1}$ Information Engineering College ,Zhengzhou University of Science and Technology, \\ Zhengzhou,450064, China \\ axiaoxia_0220@163.com, by_nan@126.com
}

Keywords: Gateway; Address Conversion; Protocol Translation

Abstract. In this paper, a model of translation gateway is proposed. By adopting the Microsoft intermediate driver technology, it can easily realize the communications between IPv4 network and IPv6 network.

\section{Introduction}

IPv4 can't meet the needs in many aspects such as amount of IP address, quality of service, security of information and so on, thereby IPv6 is introduced. The interim from IPv4 to IPv6, there needs two stages. At the first stage, interconnection among IPv6 networks is implemented by tunnel of IPv4 networks. At the second stage, the intercommunication between IPv6 network and IPv4 network is the key problem which can be solved by use of the widely accepted method of NAT-PT(Network Address Translation-Protocol Translation), described in RFC 2766[1,2,3]. In this paper, a model of translation gateway based on NAT-PT has been proposed, by which the communications between IPv4 network and IPv6 network based can be conveniently implemented in environment of Windows 7.

\section{Model of translation gateway}

The model of translation gateway is profiled as follow (Fig. 1). In Fig. 1, between IPv4 and IPv6 networks is the translation gateway which completes the address conversion and protocol translation when data packets pass through it. Being the application-level gateway, DNS-ALG deals with DNS message so that it could correctly reflect the information of domain name and this translation gateway is composed of three parts, including DNS-ALG, NAT-PT and an IPv4 address pool. DNS-ALG deals with DNS message, and shows the information of domain name. In order to complete address conversion, an one-to-one address mapping between IPv4 and IPv6 is set up so that each of the IPv4 addresses in the address pool is uniquely corresponded to an IPv6 host and vice versa[3,4].

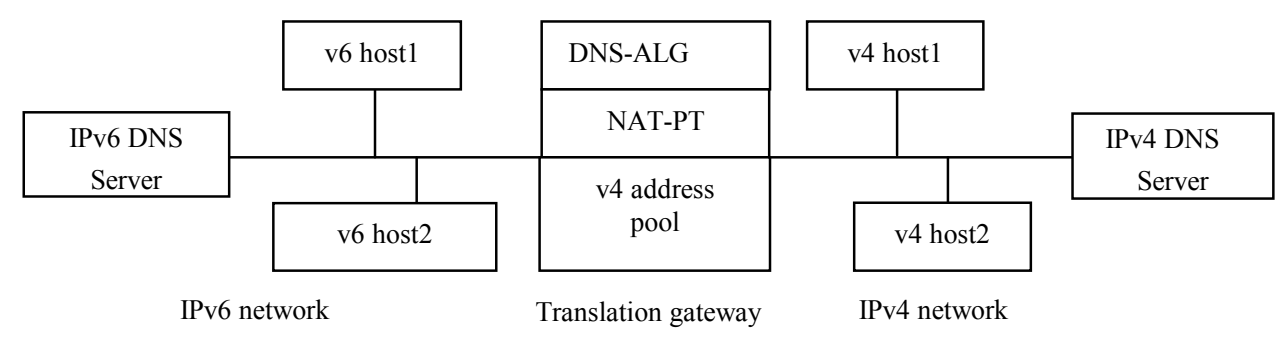

Fig.1 Model of translation gateway

\section{Main modules of translation gateway}

Address translation.Address translation is one of the most important modules in translation gateway. It aims to build and maintain the IPv4 to IPv6 address mapping. Address mapping will be built in two cases: 
(1) When an IPv6 host communicates with an IPv4 host, there is no corresponding IPv4 address to the IPv6 address, address mapping will be built.

(2) When translation gateway receives a DNS query from IPv4 realm, translation gateway will call DNS-ALG so that the DNS query from IPv4 realm could be translated into an IPv6 DNS query, and send this query to a corresponding DNS server. When the reply of DNS arrives, if there is no corresponding IPv4 mapping address to the IPv6 address in the query result, a new address mapping will be built.

Because the amount of the global unique IP address is limited, the number of hosts needing to translate is larger than the number of available IP addresses in the pool of v4 address. Once the IP addresses in the $\mathrm{v} 4$ address pool exhaust, address translation will cease to go on. In order to avoid this case, those address mappings going with the accomplished communications should be deleted from the table so that those being taken global IPv4 addresses in the pool of $\mathrm{v} 4$ address could be released for other hosts. Deleting the address mapping will be taken in two cases as below:

(1) When TCP FIN - a message of sign - arrives, the corresponding address mapping relating to the session will be deleted.

(2) Set a life span for address mapping. Once the life span is due, the corresponding address mapping will be deleted automatically.

DNS-ALG.DNS-ALG plays a crucial role in translation gateway. It is the key to carry out the bi-directional communications. By DNS-ALG, the reciprocal communications between $\mathrm{v} 4$ hosts and v6 hosts can go smoothly. Fig. 2 describes the procedures of DNS-ALG.

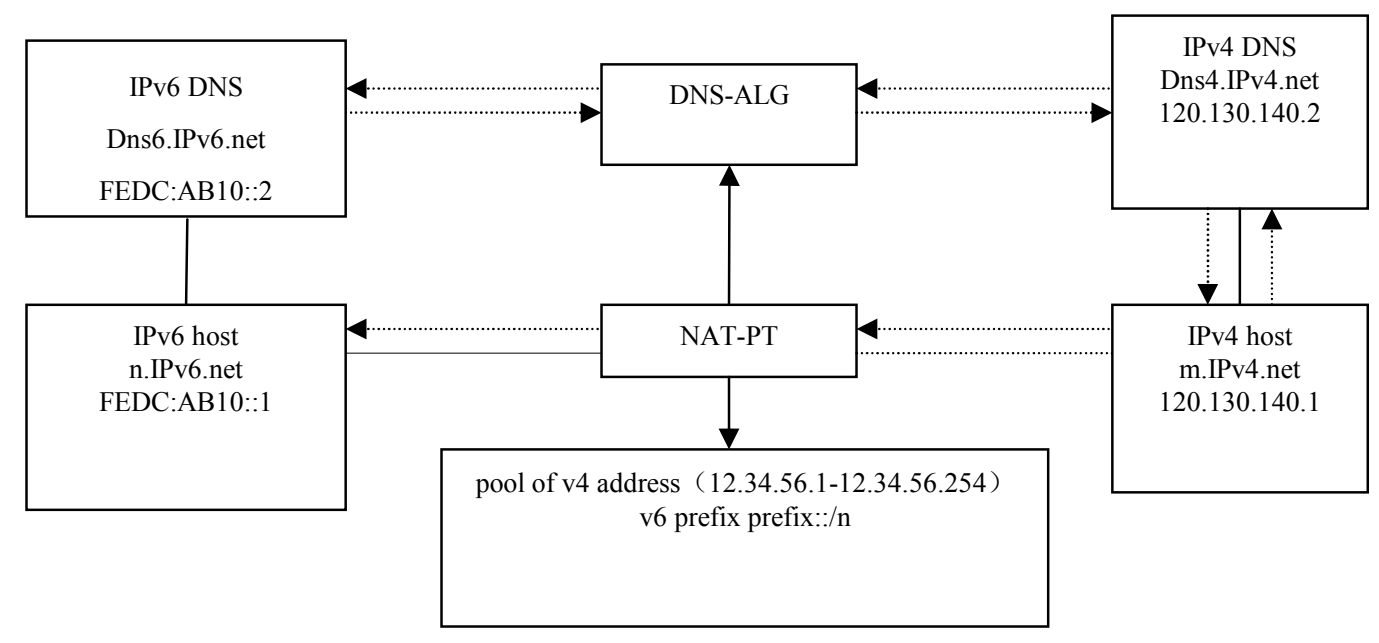

Fig. 2 The module of DNS-ALG

(1) At first, host in IPv4 network issues a DNS query to IPv4 DNS for the IPv4 address of the host in IPv6 network.

(2) IPv4 DNS looks for its records, but fails. The failure reason is mainly that IPv6 name to address mappings is held in the DNS with "AAAA" records, while IPv4 name to address mappings is held in the DNS with "A" records at the moment. Then the DNS query is delivered and intercepted by DNS-ALG.

(3) DNS-ALG translates the "A" record to "AAAA" record, and delivers it to IPv6 DNS.

(4) IPv6 DNS finds the address of IPv6 host (FEDC:AB10::1) and returns it to DNS-ALG.

(5) DNS-ALG communicates with NAT. NAT allocates a v4 address (12.34.56.1) from the pool of IPv4 address for the IPv6 address. Then address mapping is built, and the record type is modified from "AAAA" to "A". The modified record is delivered to IPv4 DNS. And IPv4 DNS returns the reply to the host in IPv4 network.

(6) The host in IPv4 network communicates with the host in IPv6 network, and sends a IPv4 packet of which source address is 120.130 .140 .1 and destination address is 12.34.56.1 to destination. 
(7) After the packet is routed to translation gateway, translation gateway searches the table of address mapping. Then the destination address is converted into FEDC:AB10::1 and source address is converted into prefix:: 120.130 .140 .1 . Subsequently, the protocol is translated.

(8) After the translations of address and protocol, this packet is send to the destination by translation gateway, then received by the host of IPv6 network.

The communications, which is initiated from hosts in v6 network, is similar to the hosts in v4 network, but direction is reverse. There also exist some differences in the procedure. The main difference is no NAT involved when the DNS queries. DNS-ALG adds a prefix before 120.130.140.1 which is an address of the IPv4 host, namely prefix::120.130.140.1, then returns it to the IPv6 host which is the initiator of communications. After the formal communications starts, the IPv6 host sends out a packet of which source address is FEDC:AB10::1 and destination address is prefix::120.130.140.1. When the packet is routed to translation gateway, translation gateway communicates with NAT. NAT allocates a global unique address 12.34.56.1 in the pool of IPv4 address to the IPv6 host and builds an address mapping. Then the source address is replaced with 12.34.56.1 and the destination address is replaced with 120.130.140.1. After the address translation completes, the protocol translation starts, and the packet is rebuilt and delivered. In the end, the IPv4 destination host whose domain name is $\mathrm{m} . \mathrm{IPv} 4$.net receives this packet. Hereto, the communications finish.

Protocol translation.Protocol Translation refers to the semantically equivalently reciprocal translation between an IPv4 packet and an IPv6 packet. Protocol translation follows SIIT[5]. Due to the potential head of fragment, at the initial stage of protocol translation, the packet should be first checked whether it has the head of fragment or not. If the head of fragment exists, translate it, then the packet.

\section{Realization of translation gateway}

In Fig. 3, there are two methods to intercept the data packets, the user mode and the kernel mode. In this paper, the kernel mode is taken to intercept the data packets. The kernel of the translation gateway as a cushion is inserted into operating system, and the key technology is implemented by the NDIS (Network Driver Interface Specification) - one intermediate drivers technology of Windows 7. The aim of using intermediate drivers is to provide some functions such as miniport drivers, encryption and authentication of data packets, etc. Through the intermediate drivers technology, the kernel of the translation gateway is inserted between the network layer and NIC (Network Interface Card) driver, which can capture the data packets from network and realize the address conversion and protocol translation easily.

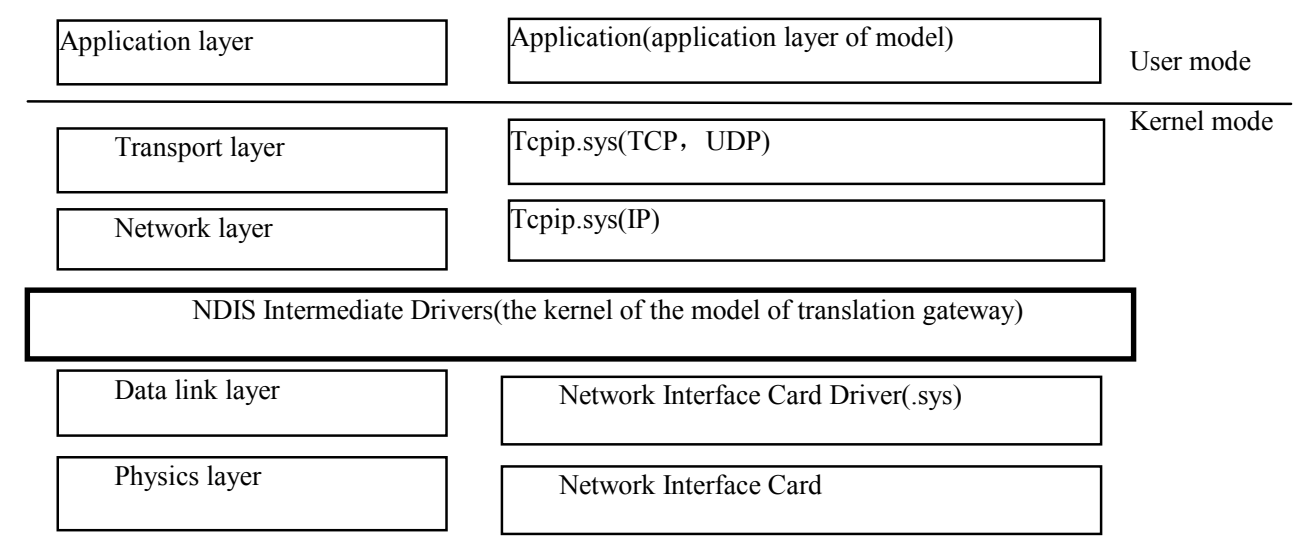

Fig. 3 The location of translation gateway in systems

Some standard functions are used in processing of the model such as DriverEntry, MiniportSend, MiniportSendPackets, ProtocolReceive, ProtocolReceivePacket, PtSendComplete, and DeviceIoControl, etc. The intermediate drivers register a Miniport interface and a Protocol interface 
for NDIS by DriverEntry, and these two interfaces include a set of well-defined standard functions respectively. As the model is being processed, these functions are rewritten.

\section{Conclusion}

the communications between the hosts for IPv4 and IPv6 is realized. Technique is presented to realize this communications under the circumstance of stabilizing the cost of network upgrade. It will helps to generalize application of IPv6.

\section{ACKNOWLEDGEMENT}

This research is supported by the Natural Science Foundation of Henan Province, China(Grant No. 122300410170).

\section{References}

[1] G.Tsirtsis, P.Srisuresh, Network Address Translation-Protocol Translation (NAT-PT). RFC 2766. (2000)

[2] P.Srisuresh, K.Egevang, Traditional IP Network Address Translator (Traditional NAT). RFC 3022. (2001)

[3] HUANG Zhi-hu, WANG Kang, Research of IPv4/IPv6 Translate Gateway based on windows[J]. Computer Engineering, 2005,31(20):138-140.

[4] ZHANG Peng, YANG Guo-yu, IPv4/IPv6 protrocol translation mechanism on backbone[J].Journal of UEST of China, 2005, 34(5):626-629.

[5] HAN Ling, DUAN Xiao-dong, LING Xiao-feng, ZENG Zhi-min, A translation gateway for IPv6 and IPv4 based on NAT/PT[J].Computer Engineering, 2004, 30(14):86-87,112. 